CERN-TH/2003-241

hep-ph/0310081

\title{
New Strategies to Extract Weak Phases from Neutral B Decays
}

\author{
Robert Fleischer \\ Theory Division, CERN, CH-1211 Geneva 23, Switzerland
}

\begin{abstract}
We discuss new, theoretically clean strategies to determine the angle $\gamma$ of the unitarity triangle from $B_{d} \rightarrow D K_{\mathrm{S}(\mathrm{L})}, B_{s} \rightarrow D \eta^{\left({ }^{\prime}\right)}, D \phi, \ldots$ decays, and point out that the $B_{s} \rightarrow D K_{\mathrm{S}(\mathrm{L})}$ and $B_{d} \rightarrow D \pi^{0}, D \rho^{0}, \ldots$ modes allow very interesting determinations of the $B_{q}^{0}-\overline{B_{q}^{0}}$ mixing phases $\phi_{s}$ and $\phi_{d}$, respectively. Their colour-allowed counterparts $B_{s} \rightarrow D_{s}^{(*) \pm} K^{\mp}, \ldots$ and $B_{d} \rightarrow D^{(*) \pm} \pi^{\mp}, \ldots$ also offer new methods to probe $\gamma$.
\end{abstract}

Invited talk at the HEP2003 Europhysics Conference

Aachen, Germany, 17-23 July 2003

To appear in the Proceedings

CERN-TH/2003-241

October 2003 



\section{Introduction}

The time-dependent CP asymmetries of neutral $B_{q}$-meson decays $(q \in\{d, s\})$ into CP eigenstates, which satisfy $(\mathcal{C P})|f\rangle= \pm|f\rangle$, provide valuable information [1]:

$$
\begin{aligned}
& \frac{\Gamma\left(B_{q}^{0}(t) \rightarrow f\right)-\Gamma\left(\overline{B_{q}^{0}}(t) \rightarrow f\right)}{\Gamma\left(B_{q}^{0}(t) \rightarrow f\right)+\Gamma\left(\overline{B_{q}^{0}}(t) \rightarrow f\right)} \\
& \quad=\frac{\mathcal{A}_{\mathrm{CP}}^{\mathrm{dir}} \cos \left(\Delta M_{q} t\right)+\mathcal{A}_{\mathrm{CP}}^{\mathrm{mix}} \sin \left(\Delta M_{q} t\right)}{\cosh \left(\Delta \Gamma_{q} t / 2\right)-\mathcal{A}_{\Delta \Gamma} \sinh \left(\Delta \Gamma_{q} t / 2\right)}
\end{aligned}
$$

Here the CP-violating observables

$$
\mathcal{A}_{\mathrm{CP}}^{\mathrm{dir}} \equiv \frac{1-\left|\xi_{f}^{(q)}\right|^{2}}{1+\left|\xi_{f}^{(q)}\right|^{2}} \quad \text { and } \quad \mathcal{A}_{\mathrm{CP}}^{\mathrm{mix}} \equiv \frac{2 \operatorname{Im} \xi_{f}^{(q)}}{1+\left|\xi_{f}^{(q)}\right|^{2}}
$$

originate from "direct" and "mixing-induced" CP violation, respectively, and are governed by

$$
\xi_{f}^{(q)}=-e^{-i \phi_{q}}\left[\frac{A\left(\overline{B_{q}^{0}} \rightarrow f\right)}{A\left(B_{q}^{0} \rightarrow f\right)}\right]
$$

where

$$
\phi_{q} \stackrel{\mathrm{SM}}{=} 2 \arg \left(V_{t q}^{*} V_{t b}\right)=\left\{\begin{array}{cc}
+2 \beta & (q=d) \\
-2 \lambda^{2} \eta & (q=s)
\end{array}\right.
$$

is the CP-violating weak $B_{q}^{0}-\overline{B_{q}^{0}}$ mixing phase. The width difference $\Delta \Gamma_{q}$, which may be sizeable in the $q=s$ case, offers another observable $\mathcal{A}_{\Delta \Gamma}$, which is, however, not independent from those in (2), and can be extracted from the following "untagged" rates:

$$
\begin{aligned}
& \left\langle\Gamma\left(B_{q}(t) \rightarrow f\right)\right\rangle \equiv \Gamma\left(B_{q}^{0}(t) \rightarrow f\right)+\Gamma\left(\overline{B_{q}^{0}}(t) \rightarrow f\right) \\
& \propto\left[\cosh \left(\Delta \Gamma_{q} t / 2\right)-\mathcal{A}_{\Delta \Gamma} \sinh \left(\Delta \Gamma_{q} t / 2\right)\right] e^{-\Gamma_{q} t}
\end{aligned}
$$

\section{$2 \quad B_{d} \rightarrow D K_{\mathrm{S}(\mathrm{L})}, B_{s} \rightarrow D \eta^{\left({ }^{\prime}\right)}, D \phi, \ldots$ and $B_{s} \rightarrow$ $D K_{\mathrm{S}(\mathrm{L})}, B_{d} \rightarrow D \pi^{0}, D \rho^{0}, \ldots$}

Let us consider in this section $B_{q}^{0} \rightarrow D^{0} f_{r}$ transitions, where $r \in\{s, d\}$ distinguishes between $b \rightarrow D s$ and $b \rightarrow D d$ processes [2, 3]. If we require $(\mathcal{C P})\left|f_{r}\right\rangle=\eta_{\mathrm{CP}}^{f_{r}}\left|f_{r}\right\rangle, B_{q}^{0}$ and $\overline{B_{q}^{0}}$ mesons may both decay into $D^{0} f_{r}$, thereby leading to interference effects between $B_{q}^{0}-\overline{B_{q}^{0}}$ mixing and decay processes, which involve the weak phase $\phi_{q}+\gamma$ :

- For $r=s$, i.e. $B_{d} \rightarrow D K_{\mathrm{S}(\mathrm{L})}, B_{s} \rightarrow D \eta^{\left({ }^{\prime}\right)}, D \phi, \ldots$, these effects are governed by a hadronic parameter $x_{f_{s}} e^{i \delta_{f_{s}}} \propto R_{b} \approx 0.4$, and are hence favourably large.

- For $r=d$, i.e. $B_{s} \rightarrow D K_{\mathrm{S}(\mathrm{L})}, B_{d} \rightarrow D \pi^{0}, D \rho^{0} \ldots$, these effects are tiny because of $x_{f_{d}} e^{i \delta_{f_{d}}} \propto-\lambda^{2} R_{b} \approx-0.02$. 


\section{$2.1 \quad B_{d} \rightarrow D K_{\mathrm{S}(\mathrm{L})}, B_{s} \rightarrow D \eta^{\left({ }^{\prime}\right)}, D \phi, \ldots$}

Let us first focus on $r=s$. If we make use of the CP eigenstates $D_{ \pm}$of the neutral $D$-meson system satisfying $(\mathcal{C P})\left|D_{ \pm}\right\rangle= \pm\left|D_{ \pm}\right\rangle$, we obtain additional interference effects between $B_{q}^{0} \rightarrow D^{0} f_{s}$ and $B_{q}^{0} \rightarrow \overline{D^{0}} f_{s}$ at the decay-amplitude level, which involve $\gamma$. The most straightforward observable we may measure is the "untagged" rate

$$
\begin{aligned}
& \left\langle\Gamma\left(B_{q}(t) \rightarrow D_{ \pm} f_{s}\right)\right\rangle \equiv \\
& \Gamma\left(B_{q}^{0}(t) \rightarrow D_{ \pm} f_{s}\right)+\Gamma\left(\overline{B_{q}^{0}}(t) \rightarrow D_{ \pm} f_{s}\right) \\
& \stackrel{\Delta \Gamma_{q}=0}{=}\left[\Gamma\left(B_{q}^{0} \rightarrow D_{ \pm} f_{s}\right)+\Gamma\left(\overline{B_{q}^{0}} \rightarrow D_{ \pm} f_{s}\right)\right] e^{-\Gamma_{q} t} \\
& \quad \equiv\left\langle\Gamma\left(B_{q} \rightarrow D_{ \pm} f_{s}\right)\right\rangle e^{-\Gamma_{q} t},
\end{aligned}
$$

providing the following "untagged" rate asymmetry:

$$
\Gamma_{+-}^{f_{s}} \equiv \frac{\left\langle\Gamma\left(B_{q} \rightarrow D_{+} f_{s}\right)\right\rangle-\left\langle\Gamma\left(B_{q} \rightarrow D_{-} f_{s}\right)\right\rangle}{\left\langle\Gamma\left(B_{q} \rightarrow D_{+} f_{s}\right)\right\rangle+\left\langle\Gamma\left(B_{q} \rightarrow D_{-} f_{s}\right)\right\rangle} .
$$

Interestingly, already this quantity offers valuable information on $\gamma$, since bounds on this angle are implied by

$$
|\cos \gamma| \geq\left|\Gamma_{+-}^{f_{s}}\right|
$$

Moreover, if we fix the sign of $\cos \delta_{f_{s}}$ with the help of the factorization approach, we obtain

$$
\operatorname{sgn}(\cos \gamma)=-\operatorname{sgn}\left(\Gamma_{+-}^{f_{s}}\right)
$$

i.e. we may decide whether $\gamma$ is smaller or larger than $90^{\circ}$. If we employ, in addition, the mixing-induced observables $S_{ \pm}^{f_{s}} \equiv \mathcal{A}_{\mathrm{CP}}^{\mathrm{mix}}\left(B_{q} \rightarrow D_{ \pm} f_{s}\right)$, we may determine $\gamma$. To this end, it is convenient to introduce the quantities

$$
\left\langle S_{f_{s}}\right\rangle_{ \pm} \equiv \frac{S_{+}^{f_{s}} \pm S_{-}^{f_{s}}}{2}
$$

Expressing the $\left\langle S_{f_{s}}\right\rangle_{ \pm}$in terms of the $B_{q} \rightarrow D_{ \pm} f_{s}$ decay parameters gives rather complicated formulae. However, complementing the $\left\langle S_{f_{s}}\right\rangle_{ \pm}$with $\Gamma_{+-}^{f_{s}}$ yields

$$
\tan \gamma \cos \phi_{q}=\left[\frac{\eta_{f_{s}}\left\langle S_{f_{s}}\right\rangle_{+}}{\Gamma_{+-}^{f_{s}}}\right]+\left[\eta_{f_{s}}\left\langle S_{f_{s}}\right\rangle_{-}-\sin \phi_{q}\right]
$$

where $\eta_{f_{s}} \equiv(-1)^{L} \eta_{\mathrm{CP}}^{f_{s}}$, with $L$ denoting the $D f_{s}$ angular momentum 2]. If we use this simple - but exact - relation, we obtain the twofold solution $\gamma=\gamma_{1} \vee \gamma_{2}$, with $\gamma_{1} \in\left[0^{\circ}, 180^{\circ}\right]$ and $\gamma_{2}=\gamma_{1}+180^{\circ}$. Since $\cos \gamma_{1}$ and $\cos \gamma_{2}$ have opposite signs, (92) allows us to fix $\gamma$ unambiguously. Another advantage of (111) is that $\left\langle S_{f_{s}}\right\rangle_{+}$and $\Gamma_{+-}^{f_{s}}$ are both proportional to $x_{f_{s}} \approx 0.4$, so that the first term in square brackets is of $\mathcal{O}(1)$, whereas the second one is of $\mathcal{O}\left(x_{f_{s}}^{2}\right)$, hence playing a minor rôle. In order to extract $\gamma$, we may also employ $D$ decays into $\mathrm{CP}$ non-eigenstates $f_{\mathrm{NE}}$, where we have to deal with complications

originating from $D^{0}, \overline{D^{0}} \rightarrow f_{\mathrm{NE}}$ interference effects [4]. Also in this case, $\Gamma_{+-}^{f_{s}}$ is a very powerful ingredient, offering an efficient, analytical strategy to include these interference effects in the extraction of $\gamma[3]$. 


\section{$2.2 \quad B_{s} \rightarrow D K_{\mathrm{S}(\mathrm{L})}, B_{d} \rightarrow D \pi^{0}, D \rho^{0}, \ldots$}

The $r=d$ case also has interesting features. It corresponds to $B_{s} \rightarrow D K_{\mathrm{S}(\mathrm{L})}, B_{d} \rightarrow$ $D \pi^{0}, D \rho^{0} \ldots$ decays, which can be described through the same formulae as their $r=s$ counterparts. Since the relevant interference effects are governed by $x_{f_{d}} \approx-0.02$, these channels are not as attractive for the extraction of $\gamma$ as the $r=s$ modes. On the other hand, the relation

$$
\eta_{f_{d}}\left\langle S_{f_{d}}\right\rangle_{-}=\sin \phi_{q}+\mathcal{O}\left(x_{f_{d}}^{2}\right)=\sin \phi_{q}+\mathcal{O}\left(4 \times 10^{-4}\right)
$$

offers very interesting determinations of $\sin \phi_{q}$ [2]. Following this avenue, there are no penguin uncertainties, and the theoretical accuracy is one order of magnitude better than in the "conventional" $B_{d} \rightarrow J / \psi K_{\mathrm{S}}, B_{s} \rightarrow J / \psi \phi$ strategies. In particular, $\phi_{s}^{\mathrm{SM}}=$ $-2 \lambda^{2} \eta$ could, in principle, be determined with a theoretical uncertainty of only $\mathcal{O}(1 \%)$, in contrast to the extraction from the $B_{s} \rightarrow J / \psi \phi$ angular distribution, which suffers from generic penguin uncertainties at the $10 \%$ level.

\section{$3 \quad B_{s} \rightarrow D_{s}^{(*) \pm} K^{\mp}, \ldots$ and $B_{d} \rightarrow D^{(*) \pm} \pi^{\mp}, \ldots$}

Let us now consider the colour-allowed counterparts of the $B_{q} \rightarrow D f_{q}$ modes discussed above, which we may write generically as $B_{q} \rightarrow D_{q} \bar{u}_{q}$ [5. The characteristic feature of these transitions is that both a $B_{q}^{0}$ and a $\overline{B_{q}^{0}}$ meson may decay into $D_{q} \bar{u}_{q}$, thereby leading

to interference between $B_{q}^{0}-\overline{B_{q}^{0}}$ mixing and decay processes, which involve the weak phase $\phi_{q}+\gamma$ :

- In the case of $q=s$, i.e. $D_{s} \in\left\{D_{s}^{+}, D_{s}^{*+}, \ldots\right\}$ and $u_{s} \in\left\{K^{+}, K^{*+}, \ldots\right\}$, these effects are favourably large as they are governed by $x_{s} e^{i \delta_{s}} \propto R_{b} \approx 0.4$.

- In the case of $q=d$, i.e. $D_{d} \in\left\{D^{+}, D^{*+}, \ldots\right\}$ and $u_{d} \in\left\{\pi^{+}, \rho^{+}, \ldots\right\}$, the interference effects are described by $x_{d} e^{i \delta_{d}} \propto-\lambda^{2} R_{b} \approx-0.02$, and hence are tiny.

We shall only consider $B_{q} \rightarrow D_{q} \bar{u}_{q}$ modes, where at least one of the $D_{q}, \bar{u}_{q}$ states is a pseudoscalar meson; otherwise a complicated angular analysis has to be performed.

It is well known that such decays allow determinations of the weak phases $\phi_{q}+\gamma$, where the "conventional" approach works as follows [6, 7]: if we measure the observables $C\left(B_{q} \rightarrow D_{q} \bar{u}_{q}\right) \equiv C_{q}$ and $C\left(B_{q} \rightarrow \bar{D}_{q} u_{q}\right) \equiv \bar{C}_{q}$ provided by the $\cos \left(\Delta M_{q} t\right)$ pieces of the time-dependent rate asymmetries, we may determine $x_{q}$ from terms entering at the $x_{q}^{2}$ level. In the case of $q=s$, we have $x_{s}=\mathcal{O}\left(R_{b}\right)$, implying $x_{s}^{2}=\mathcal{O}(0.16)$, so that this may actually be possible, although challenging. On the other hand, $x_{d}=\mathcal{O}\left(-\lambda^{2} R_{b}\right)$ is doubly Cabibbo-suppressed. Although it should be possible to resolve terms of $\mathcal{O}\left(x_{d}\right)$, this will be impossible for the vanishingly small $x_{d}^{2}=\mathcal{O}(0.0004)$ terms, so that other approaches to fix $x_{d}$ are required [6]. In order to extract $\phi_{q}+\gamma$, the mixing-induced observables $S\left(B_{q} \rightarrow D_{q} \bar{u}_{q}\right) \equiv S_{q}$ and $S\left(B_{q} \rightarrow \bar{D}_{q} u_{q}\right) \equiv \bar{S}_{q}$ associated with the $\sin \left(\Delta M_{q} t\right)$ 
terms of the time-dependent rate asymmetries must be measured, where it is convenient to introduce

$$
\left\langle S_{q}\right\rangle_{ \pm} \equiv \frac{\bar{S}_{q} \pm S_{q}}{2}
$$

If we assume that $x_{q}$ is known, we may consider

$$
\begin{aligned}
& s_{+} \equiv(-1)^{L}\left[\frac{1+x_{q}^{2}}{2 x_{q}}\right]\left\langle S_{q}\right\rangle_{+}=+\cos \delta_{q} \sin \left(\phi_{q}+\gamma\right) \\
& s_{-} \equiv(-1)^{L}\left[\frac{1+x_{q}^{2}}{2 x_{q}}\right]\left\langle S_{q}\right\rangle_{-}=-\sin \delta_{q} \cos \left(\phi_{q}+\gamma\right),
\end{aligned}
$$

yielding

$$
\sin ^{2}\left(\phi_{q}+\gamma\right)=\frac{1+s_{+}^{2}-s_{-}^{2}}{2} \pm \sqrt{\frac{\left(1+s_{+}^{2}-s_{-}^{2}\right)^{2}-4 s_{+}^{2}}{4}},
$$

which implies an eightfold solution for $\phi_{q}+\gamma$. If we fix the sign of $\cos \delta_{q}$ with the help of factorization, a fourfold discrete ambiguity emerges. Note that this assumption allows us to extract also the sign of $\sin \left(\phi_{q}+\gamma\right)$ from $\left\langle S_{q}\right\rangle_{+}$, which is of particular interest, as discussed in [5]. To this end, the factor $(-1)^{L}$, where $L$ is the $D_{q} \bar{u}_{q}$ angular momentum, has to be properly taken into account.

Let us now discuss the new strategies to explore the $B_{q} \rightarrow D_{q} \bar{u}_{q}$ modes proposed in [5]. If $\Delta \Gamma_{s}$ is sizeable, the time-dependent "untagged" rates introduced in (5)

$$
\begin{aligned}
& \left\langle\Gamma\left(B_{q}(t) \rightarrow D_{q} \bar{u}_{q}\right)\right\rangle=\left\langle\Gamma\left(B_{q} \rightarrow D_{q} \bar{u}_{q}\right)\right\rangle e^{-\Gamma_{q} t} \\
& \quad \times\left[\cosh \left(\Delta \Gamma_{q} t / 2\right)-\mathcal{A}_{\Delta \Gamma}\left(B_{q} \rightarrow D_{q} \bar{u}_{q}\right) \sinh \left(\Delta \Gamma_{q} t / 2\right)\right]
\end{aligned}
$$

and their CP conjugates provide $\mathcal{A}_{\Delta \Gamma}\left(B_{s} \rightarrow D_{s} \bar{u}_{s}\right) \equiv \mathcal{A}_{\Delta \Gamma_{\mathrm{s}}}$ and $\mathcal{A}_{\Delta \Gamma}\left(B_{s} \rightarrow \bar{D}_{s} u_{s}\right) \equiv$ $\overline{\mathcal{A}}_{\Delta \Gamma_{\mathrm{s}}}$, which yield

$$
\tan \left(\phi_{s}+\gamma\right)=-\left[\frac{\left\langle S_{s}\right\rangle_{+}}{\left\langle\mathcal{A}_{\Delta \Gamma_{\mathrm{s}}}\right\rangle_{+}}\right]=+\left[\frac{\left\langle\mathcal{A}_{\Delta \Gamma_{\mathrm{s}}}\right\rangle_{-}}{\left\langle S_{s}\right\rangle_{-}}\right],
$$

where the $\left\langle\mathcal{A}_{\Delta \Gamma_{\mathrm{s}}}\right\rangle_{ \pm}$are defined in analogy to (13). These relations allow an unambiguous extraction of $\phi_{s}+\gamma$ if we fix again the $\operatorname{sign}$ of $\cos \delta_{q}$ through factorization. Another important advantage of (18) is that we do not have to rely on $\mathcal{O}\left(x_{s}^{2}\right)$ terms, as $\left\langle S_{s}\right\rangle_{ \pm}$and $\left\langle\mathcal{A}_{\Delta \Gamma_{\mathrm{s}}}\right\rangle_{ \pm}$are proportional to $x_{s}$. On the other hand, we need a sizeable value of $\Delta \Gamma_{s}$. Measurements of untagged rates are also very useful in the case of vanishingly small $\Delta \Gamma_{q}$, since the "unevolved" untagged rates in (17) offer various interesting strategies to determine $x_{q}$ from the ratio of $\left\langle\Gamma\left(B_{q} \rightarrow D_{q} \bar{u}_{q}\right)\right\rangle+\left\langle\Gamma\left(B_{q} \rightarrow \bar{D}_{q} u_{q}\right)\right\rangle$ to CP-averaged rates of appropriate $B^{ \pm}$or flavour-specific $B_{q}$ decays.

If we keep the hadronic parameter $x_{q}$ and the associated strong phase $\delta_{q}$ as "unknown", free parameters in the expressions for the $\left\langle S_{q}\right\rangle_{ \pm}$, we obtain

$$
\left|\sin \left(\phi_{q}+\gamma\right)\right| \geq\left|\left\langle S_{q}\right\rangle_{+}\right|, \quad\left|\cos \left(\phi_{q}+\gamma\right)\right| \geq\left|\left\langle S_{q}\right\rangle_{-}\right|
$$


which can straightforwardly be converted into bounds on $\phi_{q}+\gamma$. If $x_{q}$ is known, stronger constraints are implied by

$$
\left|\sin \left(\phi_{q}+\gamma\right)\right| \geq\left|s_{+}\right|, \quad\left|\cos \left(\phi_{q}+\gamma\right)\right| \geq\left|s_{-}\right| .
$$

Once $s_{+}$and $s_{-}$are known, we may of course determine $\phi_{q}+\gamma$ through the "conventional" approach, using (16). However, the bounds following from (20) provide essentially the same information and are much simpler to implement. Moreover, as discussed in detail in [5] for several examples, the bounds following from the $B_{s}$ and $B_{d}$ modes may be highly complementary, thereby providing particularly narrow, theoretically clean ranges for $\gamma$.

Let us now further exploit the complementarity between the $B_{s}^{0} \rightarrow D_{s}^{(*)+} K^{-}$and $B_{d}^{0} \rightarrow D^{(*)+} \pi^{-}$modes. If we look at their decay topologies, we observe that these channels are related to each other through an interchange of all down and strange quarks. Consequently, the $U$-spin flavour symmetry of strong interactions implies $a_{s}=a_{d}$ and $\delta_{s}=\delta_{d}$, where $a_{s}=x_{s} / R_{b}$ and $a_{d}=-x_{d} /\left(\lambda^{2} R_{b}\right)$ are the ratios of hadronic matrix elements entering $x_{s}$ and $x_{d}$, respectively. There are various possibilities to implement these relations. A particularly simple picture emerges if we assume that $a_{s}=a_{d}$ and $\delta_{s}=\delta_{d}$, which yields

$$
\tan \gamma=-\left[\frac{\sin \phi_{d}-S \sin \phi_{s}}{\cos \phi_{d}-S \cos \phi_{s}}\right] \stackrel{\phi_{s}=0^{\circ}}{=}-\left[\frac{\sin \phi_{d}}{\cos \phi_{d}-S}\right] .
$$

Here we have introduced

$$
S=-R\left[\frac{\left\langle S_{d}\right\rangle_{+}}{\left\langle S_{s}\right\rangle_{+}}\right]
$$

with

$$
R=\left(\frac{1-\lambda^{2}}{\lambda^{2}}\right)\left[\frac{1}{1+x_{s}^{2}}\right]
$$

which can be fixed from untagged $B_{s}$ rates through

$$
R=\left(\frac{f_{K}}{f_{\pi}}\right)^{2}\left[\frac{\Gamma\left(\overline{B_{s}^{0}} \rightarrow D_{s}^{(*)+} \pi^{-}\right)+\Gamma\left(B_{s}^{0} \rightarrow D_{s}^{(*)-} \pi^{+}\right)}{\left\langle\Gamma\left(B_{s} \rightarrow D_{s}^{(*)+} K^{-}\right)\right\rangle+\left\langle\Gamma\left(B_{s} \rightarrow D_{s}^{(*)-} K^{+}\right)\right\rangle}\right] .
$$

Alternatively, we may only assume that $\delta_{s}=\delta_{d}$ or that $a_{s}=a_{d}$. Apart from features related to multiple discrete ambiguities, the most important advantage with respect to the "conventional" approach is that the experimental resolution of the $x_{q}^{2}$ terms is not required. In particular, $x_{d}$ does not have to be fixed, and $x_{s}$ may only enter through a $1+x_{s}^{2}$ correction, which can straightforwardly be determined through untagged $B_{s}$ rate measurements. In the most refined implementation of this strategy, the measurement of $x_{d} / x_{s}$ would only be interesting for the inclusion of $U$-spin-breaking effects in $a_{d} / a_{s}$. Moreover, we may obtain interesting insights into hadron dynamics and $U$-spin-breaking effects. 


\section{Conclusions}

We have discussed new strategies to explore $\mathrm{CP}$ violation through neutral $B_{q}$ decays. In the first part, we have shown that $B_{d} \rightarrow D K_{\mathrm{S}(\mathrm{L})}, B_{s} \rightarrow D \eta^{\left({ }^{\prime}\right)}, D \phi, \ldots$ modes provide theoretically clean, efficient and unambiguous extractions of $\tan \gamma$ if we combine an "untagged" rate asymmetry with mixing-induced observables. On the other hand, their $B_{s} \rightarrow D_{ \pm} K_{\mathrm{S}(\mathrm{L})}, B_{d} \rightarrow D_{ \pm} \pi^{0}, D_{ \pm} \rho^{0}, \ldots$ counterparts are not as attractive for the determination of $\gamma$, but allow extremely clean extractions of the mixing phases $\phi_{s}$ and $\phi_{d}$, which may be particularly interesting for the $\phi_{s}$ case. In the second part, we have discussed interesting new aspects of $B_{s} \rightarrow D_{s}^{(*) \pm} K^{\mp}, \ldots$ and $B_{d} \rightarrow D^{(*) \pm} \pi^{\mp}, \ldots$ decays. The observables of these modes provide clean bounds on $\phi_{q}+\gamma$, where the resulting ranges for $\gamma$ may be highly complementary in the $B_{s}$ and $B_{d}$ cases, thereby yielding stringent constraints on $\gamma$. Moreover, it is of great advantage to combine the $B_{d} \rightarrow D^{(*) \pm} \pi^{\mp}$ modes with their $U$-spin counterparts $B_{s} \rightarrow D_{s}^{(*) \pm} K^{\mp}$, allowing us to overcome the main problems of the "conventional" strategies to deal with these modes. We strongly encourage detailed feasibility studies of these new strategies.

\section{References}

[1] R. Fleischer, Phys. Rep. 370 (2002) 537.

[2] R. Fleischer, Phys. Lett. B 562 (2003) 234.

[3] R. Fleischer, Nucl. Phys. B 659 (2003) 321.

[4] B. Kayser and D. London, Phys. Rev. D 61 (2000) 116013.

[5] R. Fleischer, hep-ph/0304027, to appear in Nucl. Phys. B.

[6] I. Dunietz and R.G. Sachs, Phys. Rev. D 37 (1988) 3186 [E: D 39 (1989) 3515]; I. Dunietz, Phys. Lett. B 427 (1998) 179; D.A. Suprun et al., Phys. Rev. D 65 (2002) 054025 .

[7] R. Aleksan et al., Z. Phys. C 54 (1992) 653. 